\title{
Perceptions of Junior Faculty in General Internal Medicine Regarding Mentoring Medical Students and Residents in Scholarly Projects
}

\author{
Pinky Jha, $M D, M P H^{7}$, Brian Quinn, $M D^{7}$, Samantha Durbin, $B S^{2}$, and Sanjay Bhandari, $M D^{7}$ \\ 'Section of Hospital Medicine, Division of General Internal Medicine, Department of Medicine, Medical College of Wisconsin, Milwaukee, WI, USA; \\ ${ }^{2}$ Medical School, Medical College of Wisconsin, Milwaukee, WI, USA.
}

KEY WORDS: survey; junior faculty; mentorship; medical students; residents.

J Gen Intern Med 34(7):1098-9

DOI: $10.1007 / \mathrm{s} 11606-019-04937-4$

(c) Society of General Internal Medicine 2019

\section{BACKGROUND}

One goal of faculty development is to cultivate a culture of scholarship and mentorship. ${ }^{1}$ Scholarship is important for professional development and academic advancement and good mentors are essential for academic success. ${ }^{2}$ While mentoring is important, most clinician-educators have received little training, though are often asked to mentor students and residents. This can be difficult, given the competing challenges of their different clinical and non-clinical responsibilities. Our study's purpose was to survey junior faculty in the Division of General Internal Medicine (GIM) at a tertiary academic center to explore their perceptions regarding benefits of mentoring, barriers to mentoring, and possible interventions to counteract such barriers.

\section{METHODS}

A Qualtrics survey was emailed to all assistant professors in GIM at the Medical College of Wisconsin (MCW) to assess perceived benefits, barriers, and interventions. Responses were coded using a 5-point Likert scale ("strongly disagree," "disagree," "undecided," "agree," "strongly agree"). We dichotomized responses into positive, indicating "strongly agree" or "agree," and negative, pertaining to the remaining responses. Comparison between categorical groups was performed using a chi-square test. All analyses were performed using SAS 9.4 (Cary, NC).

\section{RESULTS}

Of 50 surveyed, we had 34 completed responses ( 30 academic hospitalists and 4 primary care physicians) corresponding to the response rate of $68 \%$. Nearly half $(47 \%)$ had been at MCW for $1-3$ years, $23 \%$ for $<1$ year, $12 \%$ for $4-6$ years, and $18 \%$

Published online March 18, 2019 for $>6$ years. Only $38 \%$ mentored resident and student projects in the first year; after that, only $50 \%$ were involved in mentoring in subsequent years (Fig. 1). Although the present involvement of the faculty in mentoring learners highly correlated with their past scholarly involvement as a student or resident (OR 2.33, CI $1.52-3.58, p=0.02$ ), only $57 \%$ of the faculty who had completed scholarly projects during their time as a student or resident subsequently mentored as faculty. The perceived benefits of mentoring included enhancing curriculum vitae (100\%), building educator portfolio (94\%), critical thinking (88\%), increased medical knowledge (85\%), enhancing scientific writing and publication (82\%), taking leadership positions in medical education ( $82 \%$ ), networking and collaboration $(79 \%)$, and meeting teaching requirements $(76 \%)$. Various barriers to mentoring are summarized in Figure 2. Perceived interventions included protected time for supporting mentorship and scholarship (97\%), incentives like financial or recognition (85\%), institutional support (82\%), coordinating with experts (79\%), training and workshops (77\%), structured peer mentorship program $(71 \%)$, and greater leadership support $(68 \%)$.

\section{DISCUSSION}

Our findings show that the majority of junior faculty have not mentored student or resident scholarly projects (case reports,

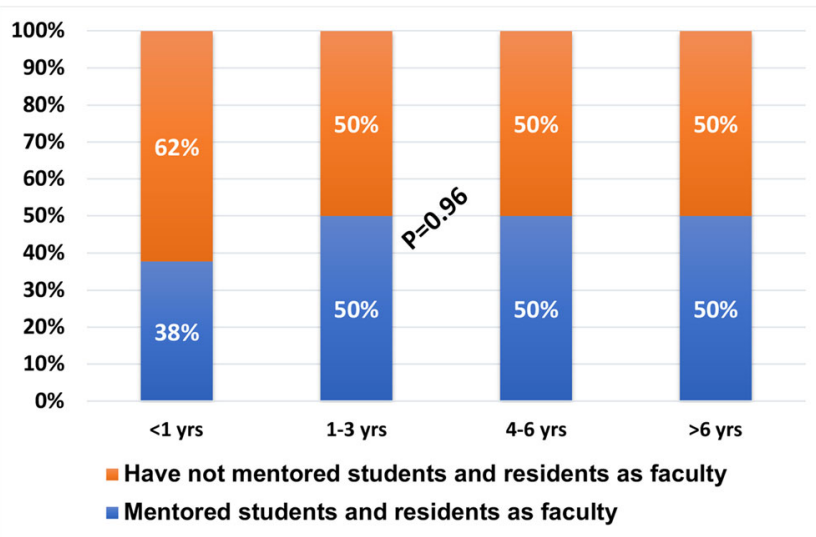

Figure 1 The faculty involvement in mentoring medical students and residents versus their time (number of years) spent as a faculty at the present institution. 


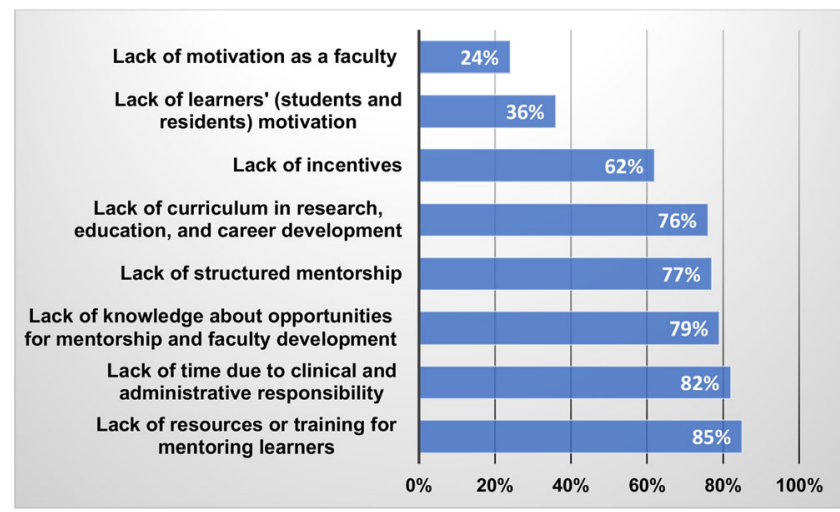

Figure 2 Various perceived barriers to mentoring medical students and residents.

quality improvement, clinical and medical education research), despite having previously completed scholarly projects during their time in medical school or residency. Further, the increase in number of years spent as faculty did not result in increased involvement in mentoring. Lack of accessible resources and formal training, lack of time due to other responsibilities, lack of institutional and leadership support, and lack of faculty development and structured mentorship programs were identified as the major barriers to mentoring learners.

Although mentoring provides various benefits to physicians in an academic setting including fulfilling their scholarly requirement, various barriers exist to which there are possible solutions. Leadership can take an active role in making the needed resources accessible, providing training opportunities and creating institutional champions/groups for supporting mentorship and scholarship. Linking senior faculty and fulltime researchers with junior faculty, along with recognizing or awarding exemplary mentorship collaborations can be integral to fostering scholarship and mentorship. ${ }^{3}$ A peer-group mentoring, which our division has currently adopted, can streamline individual efforts to increase scholarship with the formation of different peer groups based on shared interests. In contrast to a traditional mentoring group with a senior faculty as a mentor, it augments collegiality with an absence of power differential. ${ }^{4}$ Providing focused protected time may lead to increased scholarly productivity. ${ }^{5}$ Faculty development training programs, including workshops on mentoring learners in scholarly writings, are probably necessary. Aligning the interests of both learners and faculty alike enriches scholarship as a shared endeavor. The results from these interventions, however, need to be further assessed in the future to determine their effectiveness. Our survey suggests a need for multifaceted interventions to increase scholarship among junior faculty and address various barriers to their involvement in mentoring learners.

Acknowledgments: The authors wish to thank Vesna Kabic for her support in conducting the survey.

Corresponding Author: Pinky Jha, MD, MPH; Section of Hospital Medicine, Division of General Internal Medicine, Department of Medicine, Medical College of Wisconsin, HUB for Collaborative Medicine, 7th Floor, 8701 W. Watertown Plank Road, Milwaukee, WI 53226, USA (e-mail: pjha@mcw.edu).

\section{Compliance with Ethical Standards:}

Conflict of Interest: The authors declare that they do not have a conflict of interest.

Publisher's Note: Springer Nature remains neutral with regard to jurisdictional claims in published maps and institutional affiliations.

\section{REFERENCES}

1. Reid MB, Misky GJ, Harrison RA, Sharpe B, Auerbach A, Glasheen JJ. Mentorship, productivity, and promotion among academic hospitalists. J Gen Intern Med. 2012;27(1):23-7.

2. Hazzard WR. Mentoring across the professional lifespan in academic geriatrics. J Am Geriatr Soc. 1999;47(12):1466-70.

3. Kibbe MR, Pellegrini CA, Townsend CM Jr, Helenowski IB, Patti MG. Characterization of Mentorship Programs in Departments of Surgery in the United States. JAMA Surg. 2016;151(10):900-6.

4. Pololi L, Knight S. Mentoring faculty in academic medicine. A new paradigm? J Gen Intern Med. 2005;20(9):866-70.

5. Reader S, Fornari A, Simon S, Townsend J. Promoting Faculty Scholarship - An evaluation of a program for busy clinician-educators. Can Med Educ J. 2015;6(1):e43-60. 